Proceedings of the

International Geometry Center

Vol. 13, no. 2 (2020) pp. 50-67

\title{
Topology of optimal flows with collective dynamics on closed orientable surfaces
}

\author{
Alexandr Prishlyak, Maria Loseva
}

\begin{abstract}
We consider flows on a closed orientable surface with one or more heteroclinic cycles which divide the surface into two regions. One of the regions has a gradient dynamics, like Morse fields. The other region has a Hamiltonian dynamics generated by the skew gradient vector field of some simple Morse function with respect to some non-zero 2-form. We construct a complete topological invariant of the flow using the Reeb and OshemkovSharko graphs and study its properties. We also describe all possible structures of optimal flows with collective dynamics on oriented surfaces of genus $\leqslant 2$, both for flows containing center points and for flows without them.
\end{abstract}

Анотація. Розглядаються потоки на замкненій орієнтовній поверхні, які мають нерухомі точки 4-х типів: джерела, гіперболічні сідла, стоки та центри. Крім того вони мають один або декілька гетероклінічних циклів, що розбивають поверхню на дві області. Одна з областей має градієнтну динаміку, як у полів Морса. Інша область має гамільтонову динаміку, породжену полем косого градієнта простої функції Морса. Побудовано повний топологічний інваріант потоку з використанням графів Ріба для гамільтонової області та трикольорового графа Ошемкова-Шарка для градієнтної області. Досліджено властивості цього інваріанта і на його основі отримано топологічну класифікація потоків з колективною динамікою. Далі досліджуються питання, коли потік є оптимальним, тобто має найменше число нерухомих точок. В роботі описано всі можливі структури оптимальних потоків з колективною динамікою на орієнтовних поверхнях роду $\leqslant 2$ як для потоків, що містять центр, так і для потоків без нього. Також наведено сепаратрисні діаграми та 4-кольорові графи цих структур.

2010 Mathematics Subject Classification: 37c10, 37c15, 37c20

UDC 517.956.4

Keywords: Morse flow, topological equivalence, heteroclinic cycles, graph

Ключові слова: потік Морса, топологічна еквівалентність, гетероклінічні цикли, граф

DOI: http://dx.doi.org/10.15673/tmgc.v13i2.1731 
A smooth function $f$ on an orientable surface endowed with an everywhere non-zero 2-form $\omega$ generates two vector fields: the gradient field $\operatorname{grad} f$ and the skew gradient field $\operatorname{sgrad} f$ with respect to $\omega$. The trajectories of sgrad $f$ belong to the level sets of the function, and the gradient field is transversal to them. These fields have different structures and dynamics. The corresponding flows are called gradient and Hamiltonian. We consider such dynamic systems where both of these structures are presented. A flow will be said to have a collective dynamics if there is one or several heteroclinic cycles (which we will call distinguished) that divide the surface into two regions, in one of which there is a gradient dynamic and another region has a Hamiltonian dynamic. Distinguished cycles are the boundary of each of these regions. See example on the 2-torus in Figure 0.1.

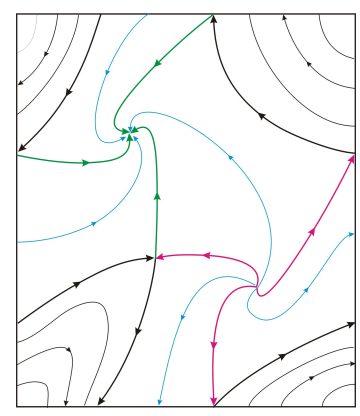

Figure 0.1. A flow with collective dynamics on the torus

In [2], this and other dynamical systems with collective dynamics were studied under certain conditions on their symmetry and analytical properties. During bifurcation, these conditions can be violated, so it is important to know the possible structures of such systems without the above conditions.

Two vector fields $X_{1}$ and $X_{2}$ on manifolds $M_{1}$ and $M_{2}$ correspondingly are called topologically equivalent, whenever there exists a homeomorphism $h: M_{1} \rightarrow M_{2}$ which maps trajectories of $X_{1}$ into trajectories of $X_{2}$ preserving orientation of those trajectories. It is also said sometimes that such vector fields have the same structure.

On closed surfaces, Morse-Smale flows form an open everywhere dense subset of the space of all vector fields. In addition, these flows are structurally stable on manifolds of an arbitrary dimension. Among flows, whose sets of non-wandering points consist of finitely many trajectories, only Morse-Smale flows are structurally stable.

M. Peixoto [9], A. Oshemkov and V. Sharko [8] and others obtained a structural classification of Morse-Smale vector fields on closed surfaces. 
A Morse-Smale flow without closed orbit is called a Morse flow. We say that a flow from some class $\mathcal{X}(M)$ of flows on a surface $M$ is optimal if it has the least number of fixed points among all flows from $\mathcal{X}(M)$. A Morse flow on a closed surface is optimal if and only if it has only one sink and one source [5]. Such a flow is also called a polar Morse flow. The topological structure of polar (optimal) Morse flows on closed surfaces was described in $[3,10,4,5]$. It is convenient to use chord diagrams as complete topological invariants of polar Morse flows. Morse-Smale flows with singularities on a surface with boundary were investigated in $[7,13$, $12,14]$.

Since the trajectories of the Hamiltonian vector field sgrad $f$ are contained in the levels sets of $f$, in order to define such vector field (up to topological equivalence) we need to know only the direction on that vector field. In fact, an ordered pair consisting of vector $\operatorname{sgrad} f$ and a $\operatorname{transversal}$ vector corresponding to the direction of increasing of the function uniquely determines an orientation of the Hamiltonian region.

A topological classification of Hamiltonian vector fields on orientable surfaces is similar to classifications of functions. Classical papers in this topic are $[15,6,16,1,11]$.

Our purpose is to construct a graph being is a complete topological invariant of a flow with collective dynamics.

\section{Simple flows With COllective DyNAmics}

In what follows, by a function $f$ on a surface $M$ we will mean a simple Morse function, i.e. a smooth function such that all of whose critical points are non-degenerated and $f$ takes distinct values at distinct critical points).

A flow on a closed surface will be called a simple flow with collective dynamic whenever

1) each fixed point is either non-degenerate or a center point;

2) there are no trajectories connecting different saddles, except for the trajectories of certain heteroclinic cycles dividing the surface into two regions called regions with gradient and Hamiltonian dynamics respectively;

3 ) in the gradient dynamic region (and on its boundary) fixed points have one of three types: sources, sinks, saddles, and the boundary set of any trajectory consists of two fixed points;

4 ) in the Hamiltonian dynamics region (and on its boundary) fixed points are of two types: saddles and centers, all other trajectories from the interior of that region are divided into two types: closed ones and those whose limit set is a saddle. 

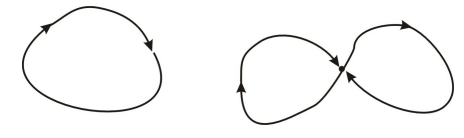

FiguRE 1.1. Trajectories in the Hamiltonian region

Thus, the dynamics of such a flow is the same as a dynamics of a gradient vector field of a Morse function of general position (Morse flow) in one of the regions and as a dynamics of a skew gradient vector field of a simple Morse function in the other region. To describe the structure of Morse flows it is convenient to use the three-color Oshemkov-Sharko' graph [8], while for the Hamiltonian flows we will use the Reeb graph [15]. If a flow has a center fixed point, then we will call a centered flow. Otherwise, when there is no centers, i.e. all fixed points are of hyperbolic type, then the flow will be called off-center flow.

\section{4-COLOR GRAPH OF A SIMPLE FLOW}

To construct the mentioned above graph we will use the separatrix flow diagram in the closure of the gradient region. In each part, into which the separatrices divide the region, we fix one regular trajectory from the source to the sink. Those trajectories divide the gradient region into curvilinear triangles and squares with the following types of sides:

(1) $s$-trajectories are separatrices going from sources to saddles;

(2) $u$-trajectories are separatrices going from the saddle to the sink;

(3) $t$-trajectories are fixed regular trajectories from source to sink;

(4) $a$-trajectories are separatrices connecting saddles belonging to the heteroclinic cycle.
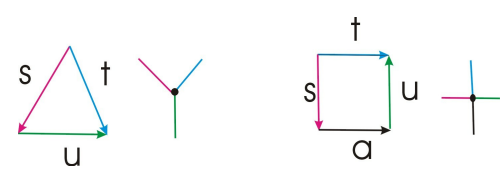

Figure 2.1. Coloring of a triangle and a square. Corresponding graphs with 3 - and 4 -vertices

The sides of each triangle are trajectories of the first three types, while the sides of each square are of all four types.

We will now define a graph being dual to the partition of the surface by the above trajectories. Its vertices correspond to triangles or squares, and the edges are trajectories of the first three types $s, u, t$ : two vertices are connected by an edge if the corresponding triangles or squares have a 
common side. The edges corresponding to the $s$-trajectory (s-edges) are colored in red, the $u$-edges in green, and the $t$-edges in blue. Thus, each vertex is incident to three edges of different colors.

For the Hamiltonian region we construct the Reeb graph by contracting each connected component of each level set of the Hamiltonian function to a point. The vertices correspond to fixed points, while each saddle point of the flow, together with the separatrices starting and finishing at that point, are contracted to a vertex of valency three. A center corresponds to the vertex of valency 1 and closed trajectories correspond to points belonging to the edges of the Reeb graph. The heteroclinic cycle also corresponds to one vertex of the graph. We connect this vertex by edges with the corresponding vertices of squares intersecting that cycle. The edges that are not colored with either of the first three colors will be colored in black. Thus, we obtain a graph whose edges are colored in 4 colors. The black edges are oriented by the direction of increasing the values of the Hamiltonian function.

Four-color graphs are called equivalent whenever there is an isomorphism of those graphs which preserves the colors of edges and also mutually either preserves or reverses orientation of all black edges.

In this definition, reversing orientation of black edges corresponds to reversing orientation of all the surface, without changing the direction of the vector field because the orientation of the Hamiltonian regions can be given by the directions of the vector field and the direction of increasing of the Hamiltonian function that correspond to direction of edges.

Theorem 2.1. Simple flows are topologically equivalent if and only if their 4-color graphs are equivalent.

Proof. Necessity. Similarly to [8], different choices of $t$-trajectories lead to isomorphic graphs. Therefore one can assume that this equivalence also takes $t$-trajectories to $t$-trajectories. The topological equivalence of flows determines the correspondence between $s-, u$ - and $t$-trajectories and the corresponding curvilinear triangles and squares.

This correspondence defines an isomorphism of dual graphs in the gradient region, while the fibered equivalence of Morse functions (their regular fibers coincide with the trajectories of the skew gradient field) defines an isomorphism of the corresponding Reeb graphs. At the same time, the graphs are correctly glued at the boundary of the Hamiltonian and gradient regions, since the vertex of the Reeb graph corresponding to the heteroclinic cycle uniquely corresponds to the set of edges incident to it in the 4-color graph, which in turn corresponds to the trajectories of this cycle in the dual graph. Thus, such a correspondence defines an isomorphism of graphs which preserves coloring and preserves or reverses orientations 
of black edges depending on whether the homeomorphism of the surface preserves or reverses orientation of the Hamiltonian region.

Sufficiency. Existence of topological equivalence of flows in the Hamiltonian region follows from the isomorphism of the Reeb graphs similarly to [1]. Also a given isomorphism of three-color graphs ensures an existence of topological equivalence on the corresponding gradient regions. Moreover, the mapping of edges corresponding to the heteroclinic cycle allows us to choose those homeomorphisms so that they coincide on this cycle and thus define a desired topological equivalence of flows on the entire surface.

Lemma 2.2. Four-color graph has the following properties.

1) There graph contains vertices of valency 4 which are incident to the edges of all 4 colors.

2) If a vertex is incident to an edge of one of the first three colors, then the edges of two other (among the first three) colors are incident to it. Such vertices have valency 3 (3-vertices) or 4 (4-vertices) and in the latter case they satisfy statement 1), see Figure 2.1.

3) 4-vertices are included in a cycle in which they alternate with 3vertices, and the edges of this cycle are colored in two colors red and green which also alternate with each other.

4) Red-green cycles without 4-vertices have length 4 (they correspond to a saddle point).

5) If we remove the edges incident to 4-vertices from the graph, then the resulting graphs (consisting of black edges only) will have vertices of valency 1 and 3 only. In this case for each vertex $v$ of valency 3 either two edges are directed inward $v$ and one edge is directed outward, or two edges are directed outward and one is directed inward $v$.

6) There are no oriented cycles consisting of black edges only.

7) All cycles in a 3-color subgraph have even lengths.

8) A 4-vertex $v$ of a three-color graph is called negative (resp. positive) if a black edge is directed inward (resp. outward) $v$. It is possible to change the orientations (of all edges of the same component) on the components of the black subgraph so that the paths in the 3-color subgraph between the vertices of the same sign have even lengths, while the paths of different signs have odd lengths.

Proof. 1) In the gradient region the trajectory from the heteroclinic cycle is the side of a curvilinear quadrilateral, which corresponds to a 4-vertex in the graph.

2) Since the edges of the first three types correspond to trajectories in the gradient region, the incidental vertices correspond to curvilinear triangles or quadrilaterals containing the sides of the first three colors. 
3) Each saddle of the heteroclinic cycle is the vertex of two curvilinear quadrilaterals and one curvilinear triangle located between them. Thus, the corresponding 3-vertex is connected to one 4-vertex by a red edge and to the other 4-vertex by a green edge. Since a curvilinear 4-gon has two vertices lying on a heteroclinic cycle, each 4 -vertex $v$ belongs to a chain of the form: 4 -vertex - 3-vertex - v-3-vertex - 4-vertex. These vertices are connected by edges of red and green color and by property 2) these colors alternate.

4) For each saddle point $w$ two red trajectories go into $w$ and two green trajectories go out from $w$. Those 4 trajectories correspond to a cycle of length 4. Consider any red edge. Then there possible two cases: a) the corresponding red separatrix goes into the saddle in the interior of the gradient region, b) this saddle belongs to a heteroclinic cycle. In the first case, it belongs to a red-green cycle of length 4 , while in the second case it belongs to a cycle with a 4 -vertex.

5) After removing the edges incident to the 4-vertices the resulting black graph will be a Reeb graph of a simple Morse function, whose vertices of valency 3 correspond to a the saddles and the vertices of valency 1 to points of local extremum (flow center) or to an edge component coinciding with the heteroclinic cycle.

6) This follows from the fact that the orientation of each black edge corresponds to the direction of increasing of the Hamiltonian function.

7) Orientations of red and green edges determine orientations of curvilinear triangles and quadrilaterals. If there is a cycle of odd length, then the union of the corresponding curvilinear triangles and quadrilaterals is a Möbius strip, since any adjacent ones in this chain have opposite orientations. This contradicts the condition of surface orientation.

8) This property is similar to the previous one and is equivalent to the agreeing of orientations on the components of the intersection of the Hamiltonian and gradient regions.

A graph whose edges are colored in 4 colors, edges of the fourth type are oriented, and for which conditions 1)-8) are satisfied will be called admissible.

Theorem 2.3. For each admissible graph there exists a simple flow on some orientable surface whose 4-color graph is equivalent to a given graph.

Proof. Similarly to Morse flows, we define a standard flow on each curvilinear triangle and square corresponding to the vertices and glue those flows in accordance with red, green, or blue edges. Using black edges as in the Reeb graph, we construct a simple Morse function on a surface with a boundary (being constant on the components of the boundary). Consider its 
skew gradient vector field. We glue the components of the boundary of the obtained surfaces by the black edges incident to the 4 -vertices and so that the directions of motion along them coincide. In a neighbourhood of the heteroclinic cycle we use the skew gradient vector field of the Morse function as a part of the Bolsinov-Fomenko atom. In neighbourhoods of saddle points in the gradient region the flow is replaced by a standard flow at the saddle point in the corresponding coordinate system at this point. One can produce the above construction to make the corresponding vector field smooth. The resulting flow is the desired one.

\section{Optimal CEnTered flows}

A centered flow (i.e. a flow having a fixed point of type center) is called optimal if it has the least number of fixed points among all centered flows on the surface.

Theorem 3.1. A flow with collective dynamics on a closed oriented surface of genus $g$ is optimal if and only if it has one source, one sink, one center, and $2 g+1$ saddle fixed points.

Proof. By definition the Hamiltonian region contains a center, while the gradient region contains a source and sink. Each of those three points has a Poincare index 1 and each saddle has index -1 . Then by the PoincareHopf theorem the sum of the indices of the vector field of saddles is less or equal than $3-(2-2 g)=2 g+1$. On the other hand, a flow with a given number and types of fixed points can be easily constructed using the optimal Morse flow (gradient field of the minimal Morse function) by introducing a loop with a center inside it in a neighbourhood of any point on a regular trajectory as in Figure 4.1.

Corollary 3.2. A simple centered flow is optimal if and only if its 4-color graph has a single red-blue cycle (it corresponds to the source), a single green-blue cycle (it corresponds to the sink), and a single vertex of valency 1 (a center point).

\section{The Structure of optimal Centered Flows on the 2-SPhere}

Theorem 4.1. On the 2-sphere all optimal flows have the same topological structure. Their separatrix diagram and the corresponding 4-color graph are shown in Figure 4.1.

Proof. It follows from Theorem 4.1 that the optimal flow on a sphere has one source, one sink, one center, and one saddle. Then the heteroclinic cycle has length 1 , so it is in fact homoclinic. Then, in addition to the trajectory 

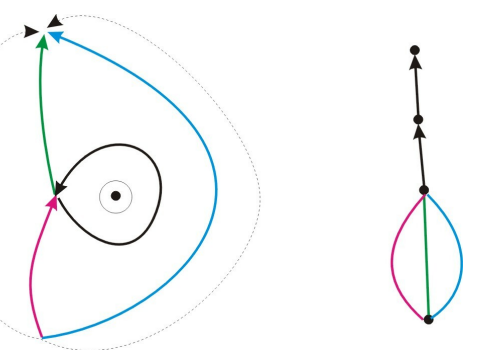

Figure 4.1. Optimal centered flow on 2-sphere

of the homoclinic cycle, the saddle also has the incoming $s$-trajectory and the outgoing $u$-trajectory.

It also follows that the gradient region is homeomorphic to a 2-disc. For any two interior points in a 2-disc there is a unique, up to a homeomorphism, possibility to join them by mutually disjoint paths with a point on the boundary. Hence, there is a unique (up to homeomorphism) disposition of $u$ - and $s$-trajectories on the sphere with respect to the homoclinic cycle.

In the gradient region the $t$-trajectory going from the source to the sink can also be selected in a unique way up to a homeomorphism. Indeed, in a neighbourhood of the source the flow is topologically equivalent to the flow $\{x, y\}$. Each regular trajectory is determined by the angle between it and the red trajectory, and a continuous change of the angle corresponds to a continuous deformation of one regular trajectory into another. This allows to construct the desired homeomorphism.

Thus in Figure 4.1, it is shown the only possible structure of the optimal centered flow on the sphere.

5. The structure of optimal Centered Flows ON THE 2-TORUS

Theorem 5.1. On the 2-torus there exists a unique, up to a topological equivalence, optimal simple centered flow with a heteroclinic cycle of length 3 (Figure 5.1), one with a heteroclinic cycle of length 2 (Figure 5.2), and one with a homoclinic cycle (Figure 5.3). Any optimal simple flow on the 2-torus has one of these three structures.

Proof. It follows from the optimality and centered conditions that the flow has three saddle points. Thus, the length of the heteroclinic cycle does not exceed 3.

First, we consider the case of flows with heteroclinic cycles of length 3 . All $s$-trajectories start in one source and finish in the saddles of that cycle. The direction of motion on the heteroclinic cycle determines the orientation of the gradient region. Enumerate $s$-trajectories in accordance with the 
orientation of the heteroclinic cycle. Consider a regular neighbourhood of a source whose boundary intersects each path outgoing from the source at a unique point. Then the orientation of this neighbourhood defines a cyclic order on the set of the intersection points of its boundary with $s$-trajectories. Let us also enumerate those points in accordance with their cyclic order so that the intersection with the first trajectory has number one. Then one can associate to each trajectory the number of its intersection point with the boundary of a neighbourhood of the source. This gives a permutation of 3 numbers, and its first element will be 1 . Thus, only two such permutations are possible: $(1,2,3)$ and $(1,3,2)$.

Evidently, a regular neighbourhood of the union of the source, $s$-trajectories, and the centered region, has three boundary components for the first permutation and one component for the second permutation. Since each such component corresponds to a sink and the optimal flow has only one sink, we see that only the substitution $(1,3,2)$ is possible. Moreover, as the constructed neighbourhood, the sink, and the trajectories outgoing from it can be located (up to a homeomorphism) in a unique way there is a unique flow structure with a heteroclinic cycle of length 3 (see Figure 5.1).
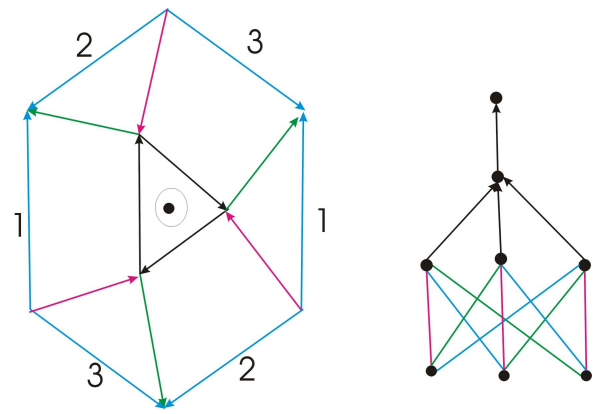

FiguRE 5.1. The centered flow with a heteroclinic cycle of length 3 on the torus

Consider flows with a heteroclinic cycle of length 2 . If we contract the Hamiltonian region to a point, we obtain a flow topologically equivalent to the optimal Morse flow on the torus. Such a flow has one source, two saddles, and one sink. Notice also that $s$-trajectories outgoing from the source can be divided into pairs so that the trajectories corresponding to one saddle enter into one pair, we see that only the partition $(1,3)(2,4)$ determine the optimal Morse flow on the torus. It can be described as a square $[-1,1] \times[-1,1]$ with glued opposite sides, a source at the vertex, (red) $s$-trajectories on the sides, and (green) $u$-trajectories at intersections 
with the coordinate axes. Due to symmetries of this flow, the inverse procedure of contracting the Hamiltonian region to a point can be carried out uniquely, up to a homeomorphism. Thus, there is only one such flow, with the structure shown in Figure 5.2.
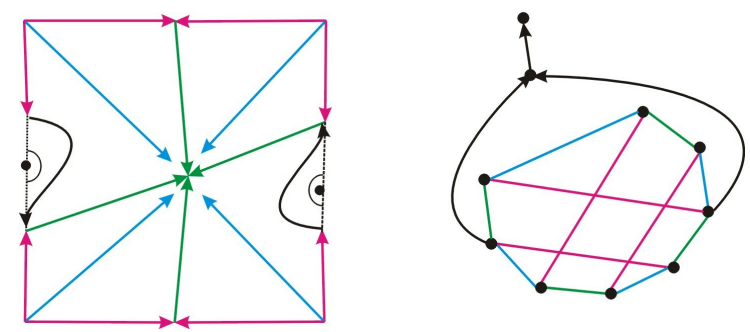

Figure 5.2. The centered flow with a heteroclinic cycle of length 2 on the torus

For a flow with a homoclinic cycle after contracting the Hamiltonian region to a point, we can similarly obtain an optimal Morse flow. A constricted point lies on a regular path. In view of the symmetries that flow can be chosen in a unique way up to a homeomorphism. Therefore, there is a single flow structure with a homoclinic cycle (Figure 5.2).
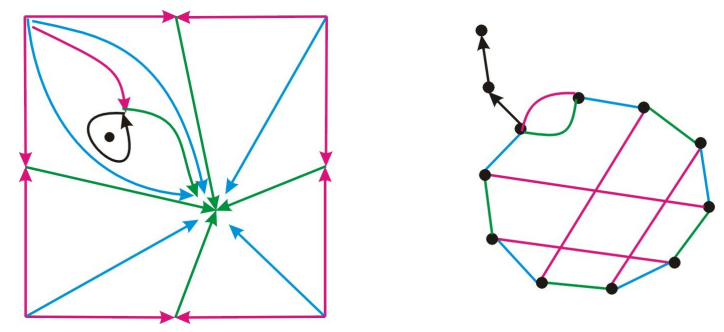

FIgURE 5.3. The centered flow with a homoclinic cycle on the torus

As the length of the heteroclinic cycle can only take values 1,2 or 3 , each optimal flow is equivalent to one of the three flows from Figures 5.1-5.3.

\section{Optimal OfF-CEnter Flows}

An off-center flow is called optimal if it has the least number of fixed points among all off-center flows on a given surface.

Theorem 6.1. A flow with off-center collective dynamics on a closed oriented surface of genus $g>0$ is optimal if and only if it has one source, one sink, and $2 g$ saddle critical point. 
Proof. As before, the source and sink are contained in the gradient region and, therefore, the number of saddles is at least $2 g$. The phase diagram of the flow with one source and one saddle on the torus is shown in Figure 8.1. Flows on surfaces of larger genus can be constructed using the operation of the connected sum of a torus with this flow and another surface with an optimal Morse flow at which regular neighbourhoods of the sink and source are removed and the flow is smoothed after gluing their boundaries.

Corollary 6.2. A simple off-center flow is optimal if and only if its 4-color graph has a single red-blue cycle (it corresponds to the source), a single green-blue cycle (it corresponds to the sink), and no vertices of valency 1 (centers).

\section{Optimal OfF-CENTER FlOWS ON THE SPHERE}

Since the off-center flow does not contain centered points, the Hamiltonian region has more than one boundary component. Each of those components corresponds to a heteroclinic cycle and contains at least one saddle. We will find the smallest possible number of singular points in the case of two boundary components and one saddle on each of them. The gradient region in this case has two components, in each of which there are a source and sink. The flow is optimal if there are no other singular points. Note that the orientation of one homoclinic cycle generates the orientation of another cycle. Sources and sinks can be unique, up to homeomorphism, defined in the components of the gradient region. Therefore, there is a single optimal flow on the sphere. Its phase portrait and 4-color graph are shown in Figure 7.1.
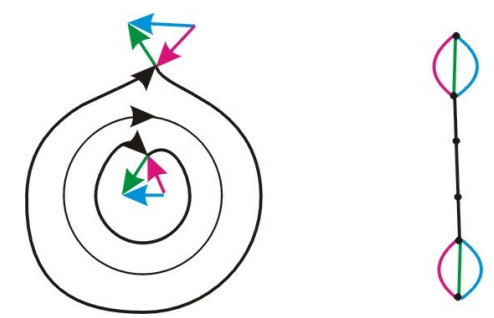

FiguRE 7.1. Optimal off-center flows on the sphere

\section{Optimal ofF-CEnter Flows on the torus}

Theorem 8.1. All optimal off-center flows on the torus are topologically equivalent. Their structure is defined by the separatrix diagram or by a 4-color graph shown in Figure 8.1. 

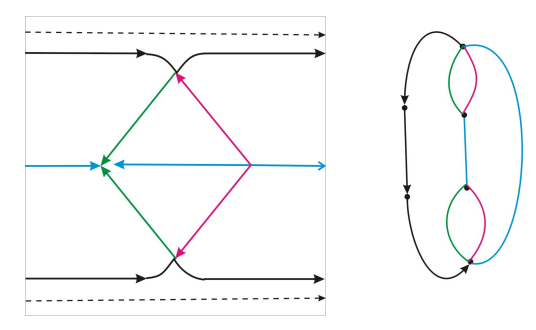

Figure 8.1. Optimal off-center flows on the torus

Proof. In accordance with the optimality criterion the Hamiltonian region has two boundary components and one saddle on each of them. Indeed, if two heteroclinic cycles have a common point (which must be a saddle), then by gluing the cylinder to the neighbourhood of this saddle, we will get a non-oriented surface as in the construction of a Klein bottle. Consider any closed trajectory in the Hamiltonian region. If this trajectory is nullhomotopic, then the Hamiltonian region divides the surface into two parts each of which has its own source and sink. Therefore, such a flow will not be optimal. Hence, the trajectory is not homotopic to zero. Cutting the surface along it, we get a cylinder. Also notice that up to a homeomorphism, the source and the trajectories connecting it with the saddles in the gradient region can be chosen uniquely.

Similarly, the same arguments hold for the sink and its separatrices. Thus, there is a single flow shown in Figure 8.1.

\section{Optimal OFF-CENTER Flows ON THE SURFACE OF GENUS 2}

We say that two flows have the same type if they have the same number of saddle points in the corresponding Hamiltonian and gradient regions as well as on their boundaries. Denote the numbers of those saddle points respectively by $h, g, d$ to denote a type, for example, type $(h 0, g 0, d 3,1)$.

Theorem 9.1. On an oriented surface of genus 2 , there are 22 topologically non-equivalent optimal off-center flows.

Proof. Each optimal off-center flow has one sink, one source, and at least 4 saddles. Moreover, the Hamiltonian region has at least 2 edge components, each of which has at least one saddle. Then the following twelve arrangements of saddles are possible.

1) Type $(h 0, g 0, d 3,1)$. This means that there are 3 saddles on one component, and 1 on the other, and there are no saddles inside the gradient and Hamiltonian regions. The Hamiltonian region is homeomorphic to a cylinder, and the gradient region is a torus with two holes $T_{2}^{2}$. Contracting 
the homoclinic cycle to a point and gluing to $T_{2}^{2}$ the heteroclinic cycle with a disk with center inside, we obtain an optimal centered flow on the torus. By Theorem 8.1 there is only one structure of such a flow. Using the symmetries of this flow, we see that the regular trajectory and the point on it (into which the homoclinic cycle is drawn) can be uniquely chosen, up to a homeomorphism. Therefore, there is only one such flow (see Figure 9.1).
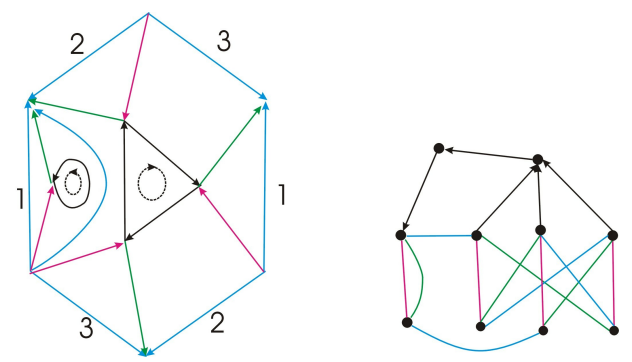

Figure 9.1. A flow of type $(h 0, g 0, d 3,1)$

2) Type $(h 0, g 0, d 2,2)$. In this case there exist two heteroclinic cycles, each of which has two saddles and there is no saddles inside the gradient and Hamiltonian regions. If we consider the gradient region and contract each of those cycles into a point (saddle), then we obtain an optimal Morse flow on the torus. The reverse procedure (for one saddle) can be carried out in two ways, which differ by the direction of orientation of the Hamiltonian cycle. Both of those methods produce topologically equivalent flows (due to the axial symmetry). The choice of the direction in the second cycle is determined by the first one due to the fact that in the Hamiltonian region (being a cylinder) the directions of movement along the bases are consistent with each other. Thus, there is only one structure of the optimal flow of this type (see Figure 9.2).
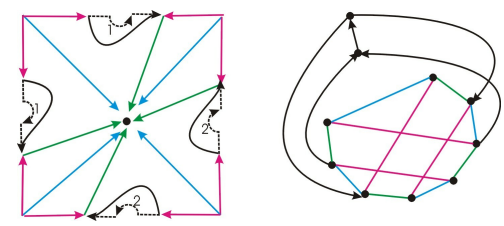

Figure 9.2. A flow of type $(h 0, g 0, d 2,2)$

Note that if heteroclinic cycles of length 2 have a common saddle point, then the union of its neighbourhood with the Hamiltonian region will be a non-orientable surface. 
3) Type $(h 0, g 1, d 2,1)$. In this case there is one saddle point in the gradient region and two heteroclinic cycles of length 2 and 1 . As before, contracting the component of the boundary of the gradient region we obtain an optimal flow on the torus. Inserting a saddle into a heteroclinic cycle of length 2 is unique up to a homeomorphism as in Figure 5.2. Creating a loop on a blue trajectory is similar to Figure 5.3. There are 4 such trajectories, but the central symmetry (as shown in Figure 5.3) implies that only two of the 4 possible options will lead to topologically non-equivalent flows. At the same time, one can introduce a loop on the blue trajectory in two ways, but only one of them is consistent with the orientations. Therefore, there are only two flows of this type.

4) Type $(h 0, g 2, d 1,1)$. There are two saddle points in the gradient region and two homoclinic cycles. As before, such flows can be obtained from the optimal flow by introducing loops (homoclinic cycles) on regular (blue) trajectories. The first loop, up to symmetries, can be introduced unambiguously as in Figure 5.3. The second loop can be created on one of the 5 blue paths and adding such a loop is unique up to a homeomorphism due to the consistency of the orientations of movements along homoclinic trajectories. One directly check that the obtained flows are topologically not equivalent. Therefore, there are 5 different topological structures of the flow of this type.

5) Type $(h 1, g 0, d 1,1,1)$. The gradient region after contracting boundary components to points gives a minimal flow on the sphere (without saddles). The choice of three regular trajectories and directions of homoclinic cycles is unambiguous up to symmetries. Therefore, there is only one topological structure of a flow of this type.

6) Type $(h 2, g 0, d 1,1)$. There are two saddles in the Hamiltonian region and two homoclinic cycles. The Hamiltonian region is a two-hole torus and the Reeb graph is the only possible Reeb graph of the optimal function on the torus. In the gradient region as in Figure 8.1, the optimal flow is unique up to equivalence. Therefore, all flows of this type have the same structure.

7) Type $(h 0,0, g 0, d 1,1,1,1)$. There are 4 homoclinic cycles. To obtain such a flow, one needs to choose two pairs of regular trajectories of the optimal flow on the sphere and the orientations agreeing on the loops on them. First consider the case when each pair includes adjacent trajectories bounding the region without two other trajectories. Then the following three cases are possible: a) the loops are directed inward the regions, b) outward, c) one pair is inward and the other one is outward the region. Now consider the case when the trajectories of one pair are opposite. Given the symmetries and consistency of the loop orientations, only one variant 
of such a flow is possible. Therefore, there are 4 topologically distinct structures of the flow of this type.

Next, we consider flows in which heteroclinic cycles have common points. Due to the consistency of orientations of such cycles there must be more than 2 such cycles. In addition, each component of the union of such cycles contains a saddle point belonging to one cycle (with red and green edges, hereinafter a simple saddle). In this case the gradient region has the genus 0 (sphere with holes) and the following types are possible.

8) Type $(h 0,0, g 0, d 4)$. All 4 heteroclinic cycles after combining give one component. There is no cycle in the type of alignment of orientations that intersects with all the remaining three cycles. Then two of them are external (intersect with one cycle), and the other two are internal (intersect with two cycles). The fourth saddle point (that does not belong to the intersection of cycles) can be either on the external or on the inner cycle. Therefore, there are two topological structures of flows of this type.

9) Type $(h 0,0, g 0, d 2,2)$. Here we have two components each having 2 cycles. The cycles are divided into pairs in accordance with the Hamiltonian regions. Simple saddles can belong either to one pair or to distinct pairs of cycles. Therefore, there are two flow patterns of this type.

10) Type $(h 0,0, g 0, d 2,1,1)$. In this case there are two cycles whose union in connected and two separated cycles (which do not intersect other cycles). The location of the red separatrix of a simple saddle from the union of the two cycles sets a cyclic order on the set of green separatrices. Therefore, there are two possibilities for it, and thus two structures of flows of this type.

11) Type $(h 0,0, g 0, d 3,1)$. Thus we have three cycles whose union is connected and one cycle separated from them. In this case, a simple saddle can be either on the outermost or on the inner loop. We have two flow structures of this type.

12) Type $(h 1, g 0, d 2,1)$. In this case the Hamiltonian region is a pants with one saddle point. Moreover, it contains two cycles with common vertex and one cycle separated from them. The orientation of the separated cycle is determined by the orientations of two others cycles. Therefore, there is only one such structure.

Thus there are 22 topologically non-equivalent optimal flows on a surface of genus 2 .

\section{Optimal Centered flows on the SURFACE OF Genus 2}

If the centered heteroclinic cycle has one or two saddles, then in order to describe the structure of such flows one can use optimal Morse flows and optimal off-center flows described above. As before, flows with a homoclinic 
cycle at the boundary of the centered region are obtained from these flows by adding a loop to the blue trajectory. In this case, depending on the orientation of this cycle, there are two ways to add that loop for each blue trajectory. Cycles with two saddles are obtained by stretching one saddle (for each saddle there are two ways to do this). After listing all flows in this way, we check them on topological equivalence.

If a centered heteroclinic cycle has three or more saddles on it, then we will describe the structure of flow using a rotation system in the source as follows. Enumerate red separatrices entering the saddles on the heteroclinic cycle with the numbers $1,2,3(4,5)$, starting from an arbitrary saddle and then going along the orientation of the cycle. The remaining (if any) saddles will be numbered with the remaining numbers (4 and 5). By sequentially entering the numbers of the saddles of the separatrices originating from the source we obtain the following flow code.

In the case of a cycle with three saddles there possible the following cases:
1) $(1,4,2,4,5,3,5)$
2) $(1,4,2,5,4,3,5)$
3) $(1,4,5,3,5,2,4)$
4) $(1,5,4,5,3,2,4)$
5) $(1,4,5,3,2,4,5)$
6) $(1,4,5,4,5,5,2)$.

Thus, there are 6 different flows of this type. In the case of a four-saddle cycle there are three different flows:
1) $(1,5,2,5,4,3)$
2) $(1,5,2,4,5,3)$
3) $(1,5,4,3,2,5)$.

If all saddle points lie on the heteroclinic cycle, then three different permutations are possible:
1) $(1,3,2,5,4)$
2) $(1,3,5,2,4)$
3) $(1,4,5,3,2)$.

\section{Conclusion}

The 4-color graphs, which we constructed, are complete topological invariants of flows with collective dynamics and give a topological classification of such flows. The methods that were used to describe all possible structures of such flows on oriented surfaces of genus 0,1 , and 2 can be used in the case of surfaces of a larger genus. Also, the resulting classification can be used to describe all possible structures of non-optimal flows. It would also be interesting to describe the bifurcation of flows with collective dynamics.

\section{REFERENCES}

[1] A. V. Bolsinov, A. T. Fomenko. Integrable Hamiltonian systems. Chapman \& Hall/CRC, Boca Raton, FL, 2004, doi: 10.1201/9780203643426. Geometry, topology, classification, Translated from the 1999 Russian original.

[2] O. A. Burilko. Collective dynamics and bifurcations in symmetric networks of phase oscillators. I. Nonlinear Oscillations, 22(2):165-195, 2019. 
[3] O. A. Giryk. Classification of polar Morse-Smale vector fields on two-dimensional manifolds. Methods Funct. Anal. Topology, 2(1):23-37, 1996.

[4] O. A. Kadubovsky. Classification of morse-smale vector fields on 2-manifolds. Visn., Mat. Mekh., Kyïv. Univ. Im. Tarasa Shevchenka, (14):85-88, 2005.

[5] Zlata Kibalko, Alexandr Prishlyak, Roman Shchurko. Trajectory equivalence of optimal Morse flows on closed surfaces. Proc. Int. Geom. Cent., 11(1):12-26, 2018, doi: $10.15673 /$ tmgc.v11i1.916.

[6] A. S. Kronrod. On functions of two variables. Uspehi Matem. Nauk (N.S.), 5(1(35)):24$134,1950$.

[7] M. V. Loseva, O. O. Prishlyak. Topology of Morse-Smale flows with singularities on the boundary of a two-dimensional disk. Proc. Int. Geom. Cent., 9(2):32-41, 2016.

[8] A. A. Oshemkov, V. V. Sharko. On the classification of Morse-Smale flows on two-dimensional manifolds. Mat. Sb., 189(8):93-140, 1998, doi: 10.1070/SM1998v189n08ABEH000341.

[9] M. M. Peixoto. On the classification of flows on 2-manifolds. In Dynamical systems (Proc. Sympos., Univ. Bahia, Salvador, 1971), pages 389-419, 1973.

[10] D. Poltavec. Equivalent polar Morse-Smale systems on two dimensional manifolds of genus 3. In Abstract of International Conference on Topology and its Applications, Kiev, page 29, 1995.

[11] A. O. Prishlyak. Topological equivalence of smooth functions with isolated critical points on a closed surface. Topology Appl., 119(3):257-267, 2002, doi: 10.1016/S01668641(01)00077-3.

[12] A. O. Prishlyak, M. V. Loseva. Optimal Morse-Smale flows with singularities on the boundary of a surface. J Math Sci, 243:279-286, 2019, doi: 10.1007/s10958-019-04539-9.

[13] O. O. Prishlyak, A. A. Prus. Morse-Smale flows on a torus with a hole. Proc. Int. Geom. Cent., 10(1):47-58, 2017.

[14] O. O. Prishlyak, A. A. Prus. A three-color graph of Morse flow and a compact surface with a boundary. Nonlinear Oscillations, 22(2):250-261, 2019, doi: 10.12697/acutm.2018.22.22.

[15] G. Reeb. Sur les points singuliers d'une forme de Pfaff complètement intégrable ou d'une fonction numérique. C. R. Acad. Sci. Paris, 222:847-849, 1946.

[16] V. V. Sharko. Functions on manifolds, volume 131 of Translations of Mathematical Monographs. American Mathematical Society, Providence, RI, 1993. Algebraic and topological aspects. Translated from the Russian by V. V. Minakhin.

Received: February 12, 2020, accepted: May 14, 2020.

Alexandr Prishlyak

Taras Shevchenko National University of Kyiv, Faculty of Mechanics and Mathematics, 4th Academician Glushkov avenue, Kyiv, 03127, Ukraine

Email: prishlyak@yahoo.com

ORCID: orcid.org/0000-0002-7164-807X

Maria Loseva

Taras Shevchenko National University of Kyiv, Faculty of computer science and Cybernetics, 4th Academician Glushkov avenue, Kyiv, 03127, Ukraine

Email: mv.loseva@gmail.com 